\title{
CLASSICAL LAGRANGE FUNCTIONS FOR THE SME*
}

\author{
N. RUSSELL \\ Physics Department, Northern Michigan University \\ Marquette, MI 49855, USA \\ E-mail: nrussell@nmu.edu
}

\begin{abstract}
A technique is presented for finding the classical Lagrange function corresponding to a given dispersion relation. This allows us to study the classical analogue of the Standard-Model Extension. Developments are discussed.
\end{abstract}

\section{Introduction}

The Standard-Model Extension, or SME, is a framework for Lorentz violation that is set up in the context of effective field theory ${ }^{1}$ in flat ${ }^{2}$ and curved $^{\sqrt{3}}$ spacetime. In the fermion sector, the SME is established by systematically adding Lorentz-breaking operators of increasing mass dimension to the Lagrange density. For the minimal SME, the dimension-three operators have coefficients $a_{\nu}, b_{\nu}, H_{\mu \nu}$, and the dimension-four operators have coefficients $c_{\mu \nu}, d_{\nu}, e_{\nu}, f_{\nu}$, and $g_{\lambda \mu \nu}$. Numerous experiments have placed bounds on these coefficients and ones from the other sectors of the SME[4]

The dispersion relation

$$
F(m, a, b, c, d, e, f, g, H ; p)=0
$$

for a mass $m$ fermion propagating in these background fields is known. ${ }^{5}$ To find it, one seeks plane-wave solutions to the modified Dirac equation. In the resulting equation, a $4 \times 4$ matrix acting on a spinor is required to vanish. The dispersion relation is the necessary condition that the matrix have zero determinant. In the limits of only $b_{\nu} \neq 0$, and only $c_{\mu \nu} \neq 0$, it takes the forms

$$
\begin{aligned}
& 0=\left(-p^{2}+b^{2}+m^{2}\right)^{2}-4(b \cdot p)^{2}+4 b^{2} p^{2} \\
& 0=p\left(\delta+2 c+c^{T} c\right) p-m^{2}
\end{aligned}
$$

*Presented at the Fifth Meeting on CPT and Lorentz Symmetry, Bloomington, Indiana, June 28-July 2, 2010 
In our conventions, light has unit speed and the metric has diagonal entries $(+1,-1,-1,-1)$. For the minimal SME, the fermion-sector dispersion relation is at most quartic in the four-momentum $p_{\nu}$. In particular, note that Eq. (2) is quartic, and that Eq. (3) is quadratic. The full function $F$ involves numerous contractions among the SME coefficients, and factorization is not straightforward $[$ [

Dispersion relations also exist for classical point particles. For a conventional particle of mass $m$, the classical Lagrange function is

$$
L=-m \sqrt{u_{\nu} u^{\nu}} .
$$

The canonical momentum is

$$
p_{\nu}=-\frac{\partial L}{\partial u^{\nu}}=\frac{m u_{\nu}}{\sqrt{u \cdot u}},
$$

and the corresponding dispersion relation is found by eliminating the fourvelocity:

$$
p^{2}=m^{2} \text {. }
$$

This proceedings contribution addresses the question of finding the classical Lagrange function corresponding to the quantum-mechanics-derived SME dispersion relation (11). Effectively, this means we are seeking a method of constructing the classical Lagrange function from a given dispersion relation.

\section{Finding the Lagrange function}

We seek a Lagrange function $L$ that yields the spacetime coordinates $x^{\nu}$ for a particle of mass $m$ as a function of a curve parameter $\lambda$. To meet the basic SME requirement of conserved energy and conserved linear momentum, $L$ cannot depend on time $x^{0}$ or position $\left(x^{1}, x^{2}, x^{3}\right)$. So, it can take the form $L=L\left(u^{\nu}, \lambda\right)$, where $u^{\nu} \equiv d x^{\nu} / d \lambda$. The trajectory is found by extremization of the action, which is the integral of $L\left(u^{\nu}, \lambda\right) d \lambda$ along a path. To ensure that the result is independent of the choice of curve parameter $\lambda, L$ must also have no explicit $\lambda$-dependence and must be homogeneous of degree one in $u^{\nu}$. Using Euler's theorem for homogeneous functions, this implies that

$$
L(u)=\frac{\partial L}{\partial u^{\nu}} u^{\nu} \equiv-p_{\nu} u^{\nu} .
$$

This equation shows that the Lagrange function can be found if the canonical momenta $p_{\nu}(u)$ are known. 
To establish a match between a classical point particle and the quantummechanical analogue of a particle, we require that the classical velocity $d x^{j} / d x^{0}$ is the same as the group velocity of a plane-wave packet. Noting that the classical velocity can be related to the four velocity in the chosen curve parametrization,

$$
\frac{d x^{j}}{d x^{0}}=\frac{d x^{j}}{d \lambda} / \frac{d x^{0}}{d \lambda}=\frac{u^{j}}{u^{0}},
$$

we find the group-velocity condition to be

$$
\frac{u^{j}}{u^{0}}=-\frac{\partial p_{0}}{\partial p_{j}}
$$

where $j$ is an index for the three cartesian spatial directions. The partial derivative on the right-hand side of Eq. (9) can be evaluated by implicit differentiation of the given dispersion relation.

We can now state a method for finding the Lagrange function for a given dispersion relation. The dispersion relation (11) and the group-velocity conditions (9) form a set of four equations in the 8 variables $u^{\mu}, p_{\nu}$; we must solve this system for the 4 variables $p_{\nu}$, in terms of the four variables $u^{\mu}$ and then substitute into Eq. (77) to get the Lagrange function.

With sufficiently small Lorentz breaking, solutions for $L$ must exist because they can only be a perturbation of the conventional ones.

An alternative is to consider the set of 5 equations (11), (7), and (9) in the 9 variables $u^{\mu}, p_{\nu}$, and $L$; use 4 of them to eliminate $p_{\nu}$, and consider the resulting polynomial equation in $L$ with $u^{\mu}$-dependent coefficients. Of the multiple solutions, only real ones can be relevant, and they correspond very roughly to particles, antiparticles, and differing spin-like states.

\section{The $b_{\nu}$ background}

As an example, we consider the case of the SME $b_{\nu}$ background. The procedure discussed above leads to an octic polynomial in $L$, expressed here in factorized form:

$$
\begin{aligned}
0= & \left(-b^{2}(b \cdot u)^{2}+b^{2} L^{2}-m^{2}(b \cdot u)^{2}\right)^{2} \times\left(b^{2} u^{2}-(b \cdot u)^{2}+\left(L+m \sqrt{u^{2}}\right)^{2}\right) \\
& \times\left(b^{2} u^{2}-(b \cdot u)^{2}+\left(L-m \sqrt{u^{2}}\right)^{2}\right) .
\end{aligned}
$$

For vanishing $b_{\nu}$, the second and third factors give the expected limits of $L$, which are Eq. (4) and its negative-mass counterpart. We disregard the first term as unphysical. The solutions for the positive-mass case are:

$$
L=-m \sqrt{u^{2}} \mp \sqrt{(b \cdot u)^{2}-b^{2} u^{2}} .
$$


The corresponding canonical momenta are

$$
p_{\nu}=\frac{m u_{\nu}}{\sqrt{u^{2}}} \pm \frac{(b \cdot u) b_{\nu}-b^{2} u_{\nu}}{\sqrt{(b \cdot u)^{2}-b^{2} u^{2}}} .
$$

This reveals that the conserved 3 -momentum $p^{j}$ is not collinear with the 3 -velocity $u^{j}$, and that the one can be nonzero when the other vanishes. Another property is the dependence of the conserved energy $E \equiv p_{0}$ on the direction of the 3 -velocity. This differs from the conventional case, where the dependence is only on the magnitude of the 3 -velocity.

Equation (12) is given in parametrization-independent form. While the proper-time interval along the curve $d \tau=\left(\eta_{\mu \nu} d x^{\mu} d x^{\nu}\right)^{1 / 2}$ can always be chosen as the curve parameter, other choices may be more convenient for different SME terms. In this case, the proper-time choice $d \lambda=d \tau$ leads to $u_{\nu} u^{\nu}=1$, and provides a convenient simplification.

\section{Spacetime torsion}

The spacetime torsion tensor, $\frac{7}{7}$ denoted $T^{\mu}{ }_{\alpha \beta}$, has a close relationship to various terms in the SME. To appreciate the rich structure underlying its 24 independent components, we may draw upon the standard $4+4+16$ irreducible decomposition with components $A^{\nu}, T_{\nu}, M_{\lambda \mu \nu}$. The axial part $A_{\nu}$ can be defined by

$$
A^{\mu} \equiv \frac{1}{6} \epsilon^{\alpha \beta \gamma \mu} T_{\alpha \beta \gamma}
$$

If torsion is present, this axial component enters the Dirac equation as a coupling to spin. It allows limits to be placed based on experiments seeking spacetime anisotropies 8 and Lorentz violation. 9

Recent work has investigated nonminimally-coupled torsion and its relation to the SME background fields. Using results from experiments testing Lorentz symmetry, the first limits were placed on 19 of the 24 independent components of the torsion tensor 10 These are measured in the standard inertial reference frame used for SME experiments. 4 The experiments involved were a dual-maser system¹011 at the Harvard-Smithsonian Center for Astrophysics, and a spin-polarized torsion pendulum ${ }^{12}$ at the University of Washington in Seattle.

Using the conventions of Ref. 10, the correspondence between axial torsion and the $b_{\nu}$ background is

$$
b_{\nu}=-\frac{3}{4} A_{\nu}
$$


Exploiting this relationship derived in the quantum-mechanical context, we may deduce from Eq. (11) the Lagrange function for a classical particle in a constant minimal-torsion background. For a point particle of mass $m$ in Minkowski spacetime, the result is

$$
L=-m \sqrt{u^{2}} \mp \frac{3}{4} \sqrt{(A \cdot u)^{2}-A^{2} u^{2}} .
$$

We remark that this Lagrange function is valid at all orders in the axial torsion tensor. We may readily deduce the dispersion relation and canonical momentum, and the results are identical to those given in Eqs. (21) and (12), up to factors of $-3 / 4$.

\section{Discussion}

Our main result is a method that, in principle, can generate the classical Lagrange function corresponding to the full minimal-SME dispersion relation. Finding the full Lagrangian is technically difficult because it involves solving a polynomial of high order. A useful illustrative example is the case of the $b_{\nu}$ background. Using this, we have deduced the Lagrange function for a point particle in the presence of minimal torsion. There are many

other avenues for investigation, some of which are discussed elsewhere 13

\section{References}

1. V.A. Kostelecký and R. Potting, Phys. Rev. D 51, 3923 (1995).

2. D. Colladay and V.A. Kostelecký, Phys. Rev. D 55, 6760 (1997); Phys. Rev. D 58, 116002 (1998).

3. V.A. Kostelecký, Phys. Rev. D 69, 105009 (2004); V.A. Kostelecký and J.D. Tasson, arXiv:1006.4106

4. Data Tables for Lorentz and CPT Violation, V.A. Kostelecký and N. Russell, 2010 edition, arXiv:0801.0287 v 3.

5. V.A. Kostelecký and R. Lehnert, Phys. Rev. D 63, 065008 (2001).

6. D. Colladay, P. McDonald, and D. Mullins, J. Phys. A 43, 275202 (2010).

7. F.W. Hehl et al., Rev. Mod. Phys. 48, 393 (1976); R.T. Hammond, Rep. Prog. Phys. 65, 599 (2002).

8. C. Lämmerzahl, Phys. Lett. B 228, 223 (1997).

9. I.L. Shapiro, Phys. Rep. 357, 113 (2002).

10. V.A. Kostelecký, N. Russell, and J.D. Tasson, Phys. Rev. Lett. 100, 111102 (2008).

11. F. Canè et al., Phys. Rev. Lett. 93, 230801 (2004).

12. B.R. Heckel et al., Phys. Rev. D 78, 092006 (2008).

13. R. Lehnert, Phys. Rev. D 68, 085003 (2003).

14. B. Altschul and D. Colladay, Phys. Rev. D 71, 125015 (2005).

15. V.A. Kostelecký and N. Russell, arXiv:1008.5062. 